\title{
The Fracture Liaison Service to close the osteoporosis care gap: a leadership educational model for undergraduate and postgraduate trainees
}

\author{
Micah $\mathrm{Yu}^{1} \cdot$ Christina Downey ${ }^{2}(\mathrm{D}) \cdot$ Karina D. Torralba ${ }^{2}$ \\ Received: 14 June 2019 / Revised: 20 September 2019 / Accepted: 23 September 2019 / Published online: 23 November 2019 \\ (C) International League of Associations for Rheumatology (ILAR) 2019
}

\begin{abstract}
Osteoporotic fractures have been rising and are a cause of severe morbidity and mortality. Care gaps exist in osteoporosis treatment and diagnosis, which presents an opportunity for education. A number of healthcare systems in the world have developed a fracture liaison service (FLS) to combat osteoporotic fractures. The Rheumatology division at Loma Linda University Health (LLUH) developed an FLS not only to address osteoporosis care gaps but to also develop a new educational model. An interdisciplinary model of osteoporosis care has been implemented along with a revamp of educational focus on osteoporosis and bone health in the rheumatology fellowship and internal medicine residency. Pre-LLUH FLS studies showed that $85 \%$ of patients pre-fracture were never screened nor treated for osteoporosis; post-fracture, only $10 \%$ of patients were treated, and only $6 \%$ had dual x-ray absorptiometry (DXA). Notably, 30\% had a prior fracture. We discuss how the FLS has served as a catalyst for education, not only at our academic center but also as an outreach for our community in order to elevate the care of osteoporosis in our community.

Key Points

- Care gaps exist in osteoporosis treatment and are addressed by the Fracture Liaison Service.

- The Loma Linda University Health Fracture Liaison Service is an interdisciplinary program.

- The Fracture Liaison Service is an educational model that gives hands on learning through an amalgam of processes, namely quality improvement through the Plan-Do-Study-Act cycle and medical education through Kolb's learning cycle and cognitive apprenticeship.
\end{abstract}

Keywords Fracture Liaison Service · Quality Improvement · Medical Education · Cognitive Apprenticeship · Experiential Learning $\cdot$ Osteoporosis $\cdot$ Fractures

Part of the Topical Collection entitled 'Empowering Medical Education to Transform: Learnings from an international perspective'

Christina Downey

cdowney@1lu.edu

Micah Yu

micyu@1lu.edu

Karina D. Torralba

ktorralba@1lu.edu

1 Division of Rheumatology, Department of Medicine, Loma Linda University Medical Center, 11234 Anderson St, Suite 1503A, Loma Linda, CA 92354, USA

2 Division of Rheumatology, Department of Medicine, Loma Linda University School of Medicine, 11234 Anderson St, Suite 1503A, Loma Linda, CA 92354, USA

\section{The osteoporosis care gap}

Osteoporosis is a common skeletal disorder defined by low bone mass that predisposes individuals to fracture, leading to significant societal burden. Over 200 million people worldwide suffer from osteoporosis with an estimated cost of $\$ 16$ billion annually in the USA, on par with breast cancer spending [1-3]. These rates are expected to rise to 3 million and $\$ 25$ billion, respectively, by 2025 [4]. The diagnosis and treatment rates of osteoporosis have lagged behind those of breast cancer screening (72.7\%) [5]. Osteoporosis is not only costly, it is also deadly. The mortality rate after a hip fracture is $79 \%$, with up to half of those patients succumbing after just 1 year $[6,7]$. Despite this, few patients are properly treated after their initial osteoporotic fracture. Fewer than $30 \%$ of postmenopausal women and less than $10 \%$ of men receive treatment after a fracture, a significant care gap $[8,9]$. By comparison, the rate of beta blocker treatment after a myocardial infarction is 
$84.4 \%$ [5]. Unfortunately, the risk of subsequent fracture in patients who have sustained a first fracture is 40-60\% [10].

Similar to global and national trends, our system discovered a similar care gap, prompting development of a Fracture Liaison Service (FLS). While the FLS has primarily served to address a dire clinical need in our community, the FLS has also proven to provide a means to provide trainees hands-on experience in quality improvement. Within the context of sound educational theory, trainees learn to effect system and cultural change. While the FLS improves the quality of care provided to patients, it also improves the quality of medical education for students, residents, and fellows.

\section{Bridging the gap: the Fracture Liaison Service}

The Fracture Liaison Service (FLS) has been established as a measure to increase the diagnosis and treatment rates of osteoporosis and to educate healthcare providers and patients, alike. It is a secondary prevention measure that aims to capture patients coming into a hospital with a fragility fracture by connecting them as part of transition of care to the outpatient resources available for long-term osteoporosis treatment. The Geisinger Health System (GHS, Danville, Pennsylvania, USA) FLS under Dr. Eric Newman and Dr. Thomas Olenginski was established by the Division of Rheumatology in 1996 and has successfully decreased hip fracture rates, increased the number of patients who receive a bone densitometry or dual-energy Xray absorptiometry (DXA) scan, and increased the number of patients who received treatment [11]. Kaiser Permanente (KP) Southern California's Healthy Bones Program under Dr. Richard O'Dell was developed in 2001 with similar successes [12]. The Glasgow Fracture Liaison Service in Scotland, developed in 1999, increased treatment rates for post-fracture patients in a cost-effective manner [13]. The Division of Rheumatology at Loma Linda University Health (LLUH), Loma Linda, California, USA drew inspiration from these examples when laying the groundwork, including its functions and processes, for its own FLS in 2016 (Fig. 1). One of the goals of the LLUH FLS is to identify clinical risk factors of secondary causes of osteoporosis. This is accomplished through a thorough history, physical exam, and laboratory testing. Another goal is to educate the patient and patient's family regarding the nature of the fracture, discuss risk factors for osteoporosis, and present the opportunity to follow up in the osteoporosis clinic for treatment. Many FLS patients, despite having sustained fracture, are surprised to learn they have osteoporosis themselves. Through a clinic coordinator, an outpatient follow-up in the osteoporosis clinic is scheduled, where therapeutic options can be discussed after the initial recovery from the fracture has occurred. Establishing care with specialized clinics in rheumatology and endocrinology redresses a well-established deficiency of primary care management of the disease [15].

\section{Steps toward change: clinical and educational contexts}

Effecting change is an uphill battle, especially when it involves well-ingrained processes. Buy-in from stakeholders, persistence, and providing practical and immediate solutions are keys. It was recognized early on, in contrast to other FLS frameworks, that the interdisciplinary nature with participation from endocrinology, geriatrics, and orthopedic surgery was key to making the LLUH FLS a success. The changes that have taken place have been over a considerable amount of time, and the steps taken were deliberate and painstaking, yet collaborative and inclusive in nature. In its infancy, many short Plan-DoStudy-Act (PDSA) cycles were implemented in order to increase the effectiveness of the service [16] (Fig. 2). The PDSA cycle, which is endorsed by the Agency for Healthcare Research and Quality (AHRQ), is a means for implementing and accelerating quality improvement, and is a means to test change in an action-oriented way.

Similar to GHS, the LLUH FLS was conceived in an academic healthcare setting, and its establishment was merged with activities that involved trainees and other medical providers. Steps in initiating and maintaining the FLS engaged trainees in this systems-based practice improvement effort, and have without a doubt factored into the success of the LLUH FLS.

\section{Steps to a solution}

Setting up the framework for a system level improvement involves multiple stakeholders and steps stemming from identifying areas of need to implementing solutions where the most benefit can be reaped. In addition, there are unexpected benefits to setting up such a framework. In the case of LLUH, it involved a complementary improvement to medical education.

\section{Identifying the problem}

Prior to implementation of the service, a gap in care was identified and quantified. The area of need was discovered when faculty from the Department of Orthopedic Surgery and Division of Rheumatology observed that patients with prior fragility fractures were re-fracturing, without having been initiated on osteoporosis treatment by primary care providers. Specialty clinics in osteoporosis, endocrinology, and rheumatology were also difficult to access due to lack of physician staffing and infrastructure. 


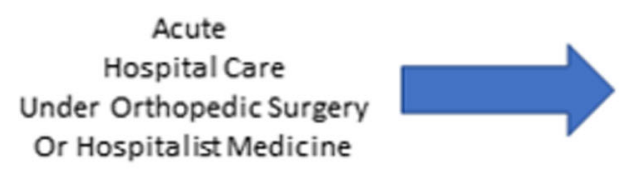

\section{Outpatient Osteoporosis Clinics \\ Rheumatology or Endocrinology: \\ Patient Care Measures}

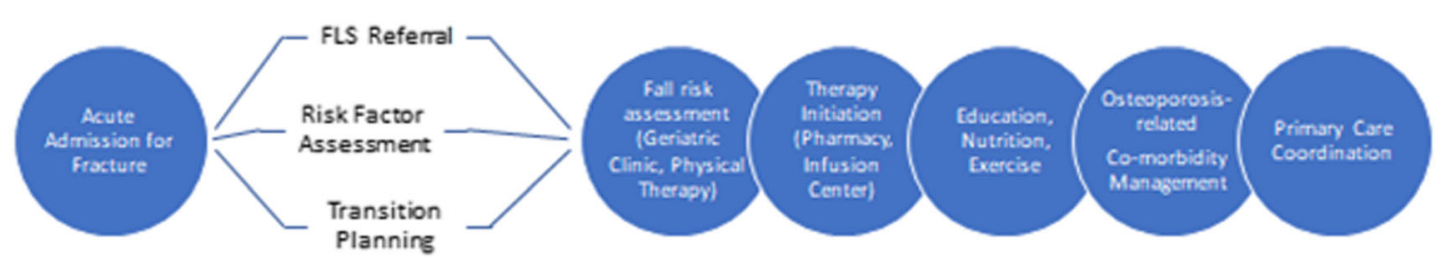

Fig. 1 Workflow for Fracture Liaison Service Loma Linda University Health. Capturing patients while admitted in the hospital through referrals from Orthopedic Surgery and Hospitalist Medicine to the FLS/ Rheumatology service is key to providing access to these patients to proper osteoporosis management on an outpatient basis. The osteoporosis outpatient clinic in turn acts as the liaison between the primary care physician, physical and occupational therapy, and orthopedic surgery. Care for an osteoporotic fracture requires a multidisciplinary team including nurses, radiologists, surgeons, pharmacists, and allied health professionals such as physical therapists, occupational therapists, and dieticians.
The first step in the process starts with a referral either by orthopedic surgery or hospital medicine while patients are hospitalized for a fragility fracture. The patients are set up for outpatient DXA scan if appropriate. Patients are triaged for follow-up either with rheumatology or endocrinology 4-6 weeks after discharge (depending on insurance, patients are booked for appointments either at the Faculty Medical Offices of Loma Linda, CA, or at the SAC Health System, which is a Federally Qualified Health Center (FQHC) in San Bernardino, California) [14]
Establishing objective data for our health system entailed health care quality research led by rheumatology faculty, internal medicine residents, and medical students. Rates of osteoporosis fracture and subsequent treatment were analyzed. Studies showed only $8.9 \%$ of patients who presented with a fragility fracture between September 2015 and February 2017 at the LLU Medical Center received osteoporotic treatment prior to the fracture; and only $10.3 \%$ received treatment after the fracture. Of the fractures analyzed, about $30 \%$ were a re-fracture event [17].

Prevention of fragility fractures is best accomplished by evaluating for osteoporosis prior to a fracture via a screening DXA scan. However, the screening and treatment rates for osteoporosis remain inadequate [18-20]. There has been a decrease in the number of facilities offering DXA screenings as well a decrease in DXA technicians [21]. Stagnant Medicare reimbursement rates for DXA services are partly to blame for these issues, apart from an overall decrease in DXAs ordered by primary care providers in general [21]. The same challenges were noted by our Internal Medicine Faculty Medical Office/Internal Medicine Department DXA unit.

Faculty FLS training Rheumatology faculty already certified by the International Society of Bone Densitometry (ISCD) obtained certification and education in FLS initiation and implementation through the National Osteoporosis Foundation
Fig. 2 PDSA cycle and Kolb's learning cycle. Key areas covered by the health quality improvement PDSA cycle mirror steps in Kolb's proposed learning cycle as part of the experiential learning experience in medical workplaces. They both are able to explain processes both involved in health improvement measure implementation and educational learning

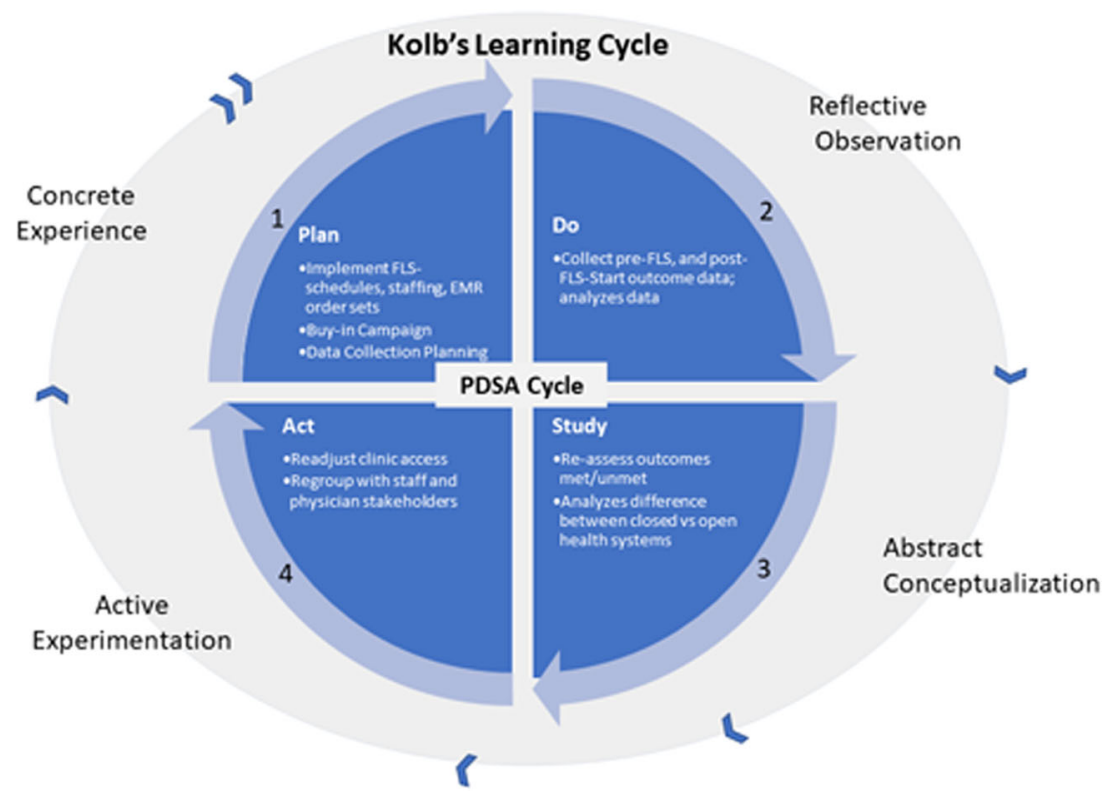


(NOF) with the support of the Department of Orthopedic Surgery.

Integrating the FLS In order to incorporate the FLS in the health system operations itself, it was vital to identify key hospital and clinic operations personnel. Meetings with electronic medical record information technology and access/call center personnel aimed to design and implement an inpatient Osteoporosis/FLS order set occurred in tandem to meetings with clinic administration to set up clinic templates and logistics. With these in place, when a fragility fracture case is admitted to the hospital, the admitting hospitalist, consulting orthopedic surgeon, or consulting neurosurgeon place a consult to the FLS service. This patient is then added to the consult list for the rheumatology service and the patient is typically seen within $24 \mathrm{~h}$.

Stakeholder buy-in, awareness campaign Recognizing that osteoporosis as a condition represents problems for patients with different or overlapping medical issues, it was important to identify key departments and faculty that were involved in the care of patients being admitted with fragility fractures. At the beginning of the project, rheumatology faculty met with various faculty and residents from Endocrinology, Orthopedic Surgery, Neurosurgery, Family Medicine, Urology, Oncology, Physical Medicine and Rehabilitation, the Outpatient Rehabilitation Center, Hospitalist Medicine, and Internal Medicine to establish buy-in for the scope of the problem, as well as inquire about barriers to providing osteoporosis screening and treatment. These meetings laid the groundwork for introduction of the concept of an FLS. An Osteoporosis Clinical Work Group was established between endocrinology, geriatrics, and orthopedic surgery to facilitate initiation and support of an FLS. Meetings were held with administrators and physicians with administrative roles in the medical center, the faculty medical group, and the medical staff association to advocate for the need to increase the number of rheumatologists and endocrinologists, and to fortify infrastructure for expansion of osteoporosis clinics into Endocrinology and Rheumatology schedules. Collaboration with endocrinology established the need to assign patients to either rheumatology or endocrinology based on the presence of comorbidities and other risk factors. For example, patients whose fracture was largely due to steroid use, or had a diagnosed or suspected rheumatic disease were assigned to rheumatology osteoporosis clinics; whereas patients with an identified endocrine disorder predisposing to osteoporosis such as a thyroid disorder, diabetes, hyperparathyroidism, and hypogonadism were liaised in endocrinology osteoporosis clinics.

Curricular change-rheumatology fellowship The rheumatology fellowship curriculum was modified to include once monthly conferences with faculty from geriatrics and endocrinology to discuss key issues in the management of osteoporosis in different populations with varied clinical conditions, including appraising guidelines set forth by the NOF, ISCD, American College of Endocrinology, American College of Gynecology, and the American College of Rheumatology (ACR). Further, learners were trained to critically analyze bone density images and results from various DXA centers, and evaluate their validity and clinical value. The inpatient consult service evolved to include consultation for osteoporosis patients. Osteoporosis was included as a key topic during interdisciplinary conferences with Physical Medicine and Rehabilitation (PM\&R).

Curricular change-residency A metabolic bone disease rotation was further introduced into the internal medicine residency curriculum, born out of a combination of resources provided by rheumatology and endocrinology, whereby residents rotating in these specialties participated in inpatient FLS consults, outpatient osteoporosis clinics, and bone density interpretation. Osteoporosis management and DXA interpretation are now part of the roster of 3-h resident clinical workshop provided by rheumatology faculty and fellows. Orthopedic surgeons and PM\&R residents are required to do a rheumatology rotation during their first year of training, allowing participation in FLS consults and osteoporosis clinics.

Community outreach Implementation of the FLS has served as a catalyst for educating the surrounding medical community. Starting in 2018, there has been an annual multi-disciplinary osteoporosis symposium showcasing different topics related to osteoporosis diagnosis and management and its relationship with other medical conditions. A wide variety of attendees have availed of these conferences including primary care and subspecialty physicians, nurse practitioners, physician assistants, pharmacists, nurses, and physical therapists.

Sustaining the change Annually, at the start of each academic year, collaborative practice conferences are held by Rheumatology faculty and fellows with Orthopedic Surgery, Family Medicine, General Medicine, and Hospitalist Medicine faculty/residents to give updates on the progress of the FLS. Fellows are asked to provide their experiences related to the initiation of an FLS consult, and the subsequent coordination of care involved to transition osteoporosis care from the acute inpatient setting to the outpatient clinic setting. Senior residents from orthopedic surgery serve as liaisons. There has also been an expansion of the number of rheumatology and endocrinology physicians and midlevel providers, justified partly by the establishment and growth of the FLS and the osteoporosis clinics. PDSA cycles are also done at each step.

Authenticating our role As of 2019, the Loma Linda University Medical Center's Executive Board gave 
recognition to the Division of Rheumatology for the establishment of a Center for Bone Health and Osteoporosis as a means to integrate research, clinical, and educational endeavors related to this field, likewise acknowledging the importance of the FLS. The International Osteoporosis Foundation is currently reviewing the LLUH FLS for certification.

\section{Outcomes}

Faculty, residents, fellows, and medical students continue to engage with outcomes projects related to care received by osteoporosis patients through FLS.

Clinical outcomes Health outcomes research continues with the help of residents, students, and fellows. Since the implementation of the FLS there has been an $8.6 \%$ increase in postfracture osteoporotic treatment within 1 year, increasing from 10 to $18.6 \%$. The LLUH FLS has also improved post-fracture DXA rates, from 6.4 to $22.4 \%$ [17, 22] (Fig. 3). In conjunction with our Breast Oncology colleagues, postmenopausal women on aromatase inhibitor therapy were also recognized as a group of patients at risk for osteoporosis, and a preliminary study showed that $69 \%$ of these patients were osteoporotic [23]. Encouragingly, there was superior adherence to bone health guidelines for breast cancer patients on aromatase inhibitors. Ninety percent of these patients were already on an appropriate dose of calcium and vitamin D, $47 \%$ obtained DXA annually, and $63.6 \%$ of patients received antiosteoporotic medication [23]. These numbers exceed those stated prior as average bone health care of the postmenopausal woman.

A number of barriers to FLS implementation were also identified. Many patients entering the LLUH FLS database were following up with outside primary care providers. Because the LLU Medical Center is the only tertiary medical center in a large geographic region, many patients come from distant locales. LLUH also operates as a largely open healthcare system as compared to KP which has a closed system, and GHS which has a hybrid system. Approximately half of patients coming in to the acute care setting have primary care providers outside of LLUH. Transitioning a patient to a set of providers outside the system and tracking if they received proper osteoporosis management became difficult. Since 2017, LLUH has embarked on an expansion of primary care departments and services (including Family Practice and General Medicine). Certain primary care providers have been identified as osteoporosis champions. It is hoped over time that there will be improvements in primary and specialty services identification of and care for patients who are at risk of having osteoporotic fractures in the future, ideally prior to developing the sentinel fracture event. The impact of interaction with the FLS team on future behavior of health professionals and the sustainability of this behavior change is a question that is currently being investigated.

As of August 2018, the LLUH Division of Rheumatology started an FLS at Riverside University Health System (RUHS) (formerly Riverside County Regional Medical Center) where its rheumatology fellows also train. This has been started in collaboration with the RUHS Department of Orthopedic Surgery and follows a similar model of steps taken to effect change as described above.

Educational outcomes Daily interaction with a quality improvement project allows trainees to see first-hand the value in evaluating practice habits and working within a system to improve those habits with tangible effects on outcomes [24]. By contributing to the FLS team while on the inpatient rheumatology service, trainees satisfy many Accreditation Council of Graduate Medical Education (ACGME) milestones for all competencies of patient care, medical knowledge, systemsbased practice, practice-based learning and improvement, professionalism, and interpersonal communication. Being part of a sustainable quality improvement project allows trainees to
Fig. 3 Osteoporotic treatment after fractures within 1 year of FLS implementtation was $18.6 \%$, verus $10 \%$ prior. In addition, receiving a DXA scan after fracture prior to FLS implementation was $6.4 \%$ whereas the rate has increased to $22.4 \%$ after $[17,22]$

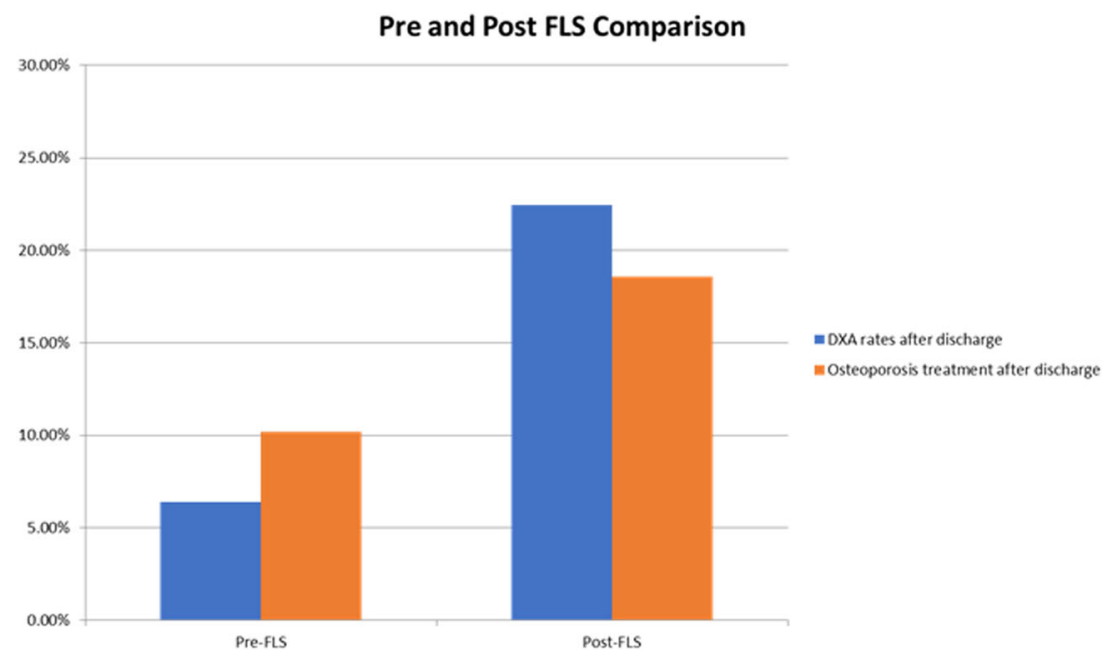


realize the large impact that working to improve the quality of care can make.

The FLS has generated scholarly work among residents and fellows, particularly in the area of health systems research, which have been presented at major meetings. Such work includes descriptions of our FLS model, improvements in DXA screening rates, and treatment initiation rates after the FLS was initiated [17, 22, 23, 25, 26]. We are in the process of linking the use of an FLS to improvements in practice habits and learning habits of trainees.

Out of five trainees who have engaged in FLS research, three have procured fellowship positions in Rheumatology, and one has secured a residency in Orthopedic Surgery. Workforce shortages are well documented in rheumatology and endocrinology. An FLS may be a way of engaging residents and students who have an interest in these fields and encouraging them to apply to fellowship positions.

\section{Medical education perspectives and the FLS}

Experiential learning is one of the key tenets by which trainees inculcate knowledge and skills. Kolb's learning cycle conceptualizes an experiential learning theory that

Table 1 Components of cognitive apprenticeship and their applicability to learning processes adopted by the physician learning how to initiate/ maintain the FLS (physician FLS lead) and those adopted by trainees (medical students, residents, fellows) participating in the FLS

\begin{tabular}{|c|c|c|}
\hline \multicolumn{2}{|c|}{ Cognitive apprenticeship } & \multirow{2}{*}{$\begin{array}{l}\text { Learning experiences provided by FLS } \\
\text { Physician FLS lead }\end{array}$} \\
\hline Component & Definition & \\
\hline Modeling & $\begin{array}{l}\text { Expert performs the skill, and learners } \\
\text { observe to build a conceptual model } \\
\text { of the processes required to } \\
\text { accomplish it. }\end{array}$ & $\begin{array}{l}\text { Takes cues and mentorship from NOF facul } \\
\text { who have launched FLS at their own } \\
\text { respective institutions. Physician attempt } \\
\text { to build up an FLS based on the experienc } \\
\text { of these faculty. }\end{array}$ \\
\hline Coaching & $\begin{array}{l}\text { Expert observes the learner performing } \\
\text { the skill and offers suggestions, } \\
\text { feedback. }\end{array}$ & $\begin{array}{l}\text { Faculty obtain suggestions from NOF facul } \\
\text { and other health systems experts. }\end{array}$ \\
\hline Scaffolding & $\begin{array}{l}\text { Learning supports (including lessons, } \\
\text { and other activities to assist the learner } \\
\text { to progress to the next level. }\end{array}$ & $\begin{array}{l}\text { Faculty obtain NOF training, continue to } \\
\text { obtain advice from outside faculty/mento } \\
\text { and eventually do things independently. } \\
\text { Lead up studies with trainees to obtain } \\
\text { outcomes of osteoporosis patients pre-FL } \\
\text { and subsequently develop a needs assess } \\
\text { ment. }\end{array}$ \\
\hline Articulation & $\begin{array}{l}\text { Learners articulate their knowledge, } \\
\text { reasoning; e.g., questioning, } \\
\text { explaining what they are doing and } \\
\text { why they do it that way. }\end{array}$ & $\begin{array}{l}\text { Faculty articulate their reasoning for } \\
\text { establishment of an FLS to different } \\
\text { stakeholders, explaining why certain } \\
\text { physicians should be concerned about } \\
\text { osteoporosis screening and treatment in } \\
\text { their own patient populations. }\end{array}$ \\
\hline
\end{tabular}

Reflection Learners critique their own performance Faculty, through trial and error, navigate health and problem-solving processes and compare these with those of an expert, another learner.

Exploration Pushes students into a mode of problem solving on their own - critical if learners are to adapt to new problems in the real world systems to determine ways to procure support for or access to an FLS or osteoporosis clinic; figure out solutions applicable to the local health settings; compare ideal goals with actual goals and outcomes

Faculty explore best means of initiating and adopting FLS to own local healthcare setting; anticipate and recognize barriers and possible solutions to optimizing FLS.

\section{LLUMC trainees*}

Trainees observe faculty who manage patients in osteoporosis clinic in terms of patient management and education; trainees during interdisciplinary meetings observe faculty who teach faculty and trainees from other disciplines about the FLS

Faculty observe and provide feedback to trainees who seeing patients in clinic (e.g., clinical skills exam) or interpret DXAs.

Trainees obtain core learning in osteoporosis management, risk assessment, DXA interpretation during symposia, monthly interdisciplinary meetings, and during consult and clinic preceptorship. Over time, they apply this knowledge to routine cases, and then to more complex cases. Eventually learners become proficient and independent.

Trainees articulate their management plans during consult and clinic preceptorship with faculty; articulate during interdisciplinary meetings how to access FLS referrals, and issues related to liaising complex cases to the correct specialty; articulate barriers and potential solutions to FLS referrals and clinic access.

Trainees assess failures to access FLS or outpatient clinics, compare access issues with that of other health systems; do a practice habits review. Make suggestions for improving access; consult with faculty and clinic personnel for individual sets of patients.

Trainees explore barriers to clinic access and possible reasons for under/over referral; reason for inappropriate referrals. Provide practical solutions or suggestions.

* Rheumatology and endocrinology residents (including those on metabolic disease rotation), rheumatology fellows

$N O F$, National Osteoporosis Foundation; DXA, dual-energy X-ray absorptiometry; FLS, Fracture Liaison Service 
comprises a four modes or stages of active learning that recognizes learners' conscious recognition and transformation of experience. The four modes are (1) concrete experience, (2) reflective observation, (3) abstract conceptualization, and (4) active experimentation [27, 28].

We would like to propose that the PDSA cycle echoes the essence of Kolb's learning cycle (Fig. 2). "Concrete Experience" is the starting point of learning wherein a learner undergoes a learning event; this is followed by "Reflective Observation" where learners making sense of experience. While not neatly fitting into the concept, these first two steps in Kolb's cycle seemingly correspond to the "Plan" and "Do" steps in the PDSA cycle, where one plans the test or observation, including a plan for collecting data, and sets aside time to analyze the data and study the results, respectively. In "Abstract Conceptualization," learners extract what they have learned from an experience, form an opinion on its significance, and assimilate this into existing knowledge; this corresponds in a way to the "Study" aspect of the PDSA cycle where one sets aside time to analyze the data and studies the results. In "Active Experimentation," learners try out what they have learned in response to further experiences; this echoes the "Do" aspect of the PDSA cycle where a provider tries out the test on a small scale.

When taken at face value, the concept of the FLS as a medium for trainees to get hands-on learning, the FLS by itself represents a learning activity that is authentic and requires interaction within a socio-cultural context - this by itself embodies the definition of "situated cognition" or "situated learning" [29]. The osteoporosis care gap provides the context by which the FLS exerts its importance and requires a specific knowledge and skill set to address a problem. The FLS provides a long-term, practical experience for physicians and trainees to be able to participate in quality improvement within a socio-cultural context, and gain expertise via "cognitive apprenticeship." Management strategies in osteoporosis and understanding what is and what makes an FLS work provide the domain knowledge (subject matter, specific procedures) while the steps in initiating and maintaining a successful FLS provide exploratory strategies for this cognitive apprenticeship environment [30].

In the traditional sense, apprenticeship is an instructional model whereby an apprentice learns an observable task or skill under the guidance of a master craftsperson. In contrast, under cognitive apprenticeship, while the task or skill itself is still observable, the emphasis is on the thinking process associated with learning the task or skill. Cognitive apprenticeship has six main components: modelling, coaching, scaffolding, articulation, reflection, and exploration [30, 31]. All of these are defined and described in the context of the FLS in Table 1. Because learning is a lifelong process, the physician lead who established the FLS also went through the same process of cognitive apprenticeship, although in a broader and less structured scale by obtaining mentorship from faculty from other institutions.

\section{Conclusion}

The FLS has been a model for trainees of care elevation through quality improvement while addressing a local and national problem. By having trainees learn about osteoporosis through the FLS, trainees are able to extract concrete learning and experience via a blended educational process via Kolb's learning cycle and a quality improvement process via a PDSA cycle. The FLS also enhances tenets of cognitive apprenticeship not only for residents and students but for faculty as well through all six tenets including modeling and coaching. Overall, the FLS is a superb model for not only a quality improvement project that works to improve a care gap, but also one that incorporates education at multiple levels, meeting multiple competencies.

\section{Compliance with ethical standards}

Disclosures None.

\section{References}

1. Blume SW, Curtis JR (2011) Medical costs of osteoporosis in the elderly medicare population. Osteoporos Int 6:1835-1844

2. International Osteoporosis Foundation. Epidemiology. https:// www.iofbonehealth.org/epidemiology. Accessed 11 April 2019

3. Hassett MJ, Elkin EB (2013) What does breast cancer treatment cost and what is it worth? Hematol Oncol Clin North Am 4:829 841

4. Burge R, Dawson-Hughes B, Solomon DH et al (2007) Incidence and economic burden of osteoporosis-related fractures in the United States, 2005-2025. J Bone Miner Res 3:465-475

5. National Committee for Quality Assurance. State of Healthcare Quality Report. http://www.ncqa.org/report-cards/health-plans/ state-of-health-care-quality/2017-table-of-contents. Accessed 17 April 2019

6. Panula J, Pihlajamäki H, Mattila VM et al (2011) Mortality and cause of death in hip fracture patients aged 65 or older: a population-based study. BMC Musculoskelet Disord 12:105

7. Schnell S, Friedman SM, Mendelson DA et al (2010) 1-year mortality of patients treated in a hip fracture program for elders. Geriatric Orthop Surg Rehabil 1:6-14

8. Nazrun AS, Tzar MN, Mokhtar SA, Mohamed IN (2014) A systematic review of the outcomes of osteoporotic fracture patients after hospital discharge: morbidity, subsequent fractures, and mortality. Ther Clin Risk Manag 10:937-948

9. National Osteoporosis Foundation. Osteoporosis Fast Facts. https:// cdn.nof.org/wp-content/uploads/2015/12/Osteoporosis-Fast-Facts. pdf. Accessed 17 April 2019.

10. Center JR, Bliuc D, Nguyen TV et al (2007) Risk of subsequent fracture after low-trauma fracture in men and women. JAMA. 297(4):387-394 
11. Newman ED (2011) Perspectives on pre-fracture intervention strategies: the Geisinger Health System Osteoporosis Program. Osteoporos Int 3:451-455

12. Dell R (2011) Fracture prevention in Kaiser Permanente Southern California. Osteoporos Int 3:457-460

13. McLellan AR, Wolowacz SE, Zimovetz EA et al (2011) Fracture Liaison Services for the evaluation and management of patients with osteoporotic fracture: a cost-effectiveness evaluation based on data collected over 8 years of service provision. Osteoporos Int 7:2083-2098

14. Camacho PM, Petak SM, Binkley N, Clarke BL, Harris ST, Hurley DL, Kleerekoper M, Lewiecki EM, Miller PD, Narula HS, PessahPollack R, Tangpricha V, Wimalawansa SJ, Watts NB (2016) American Association of Clinical Endocrinologists and American College of Endocrinology Clinical Practice Guidelines for the Diagnosis and Treatment of Postmenopausal Osteoporosis2016. Endocr Pract 22(9):1111-1118

15. Gehlbach SH, Fournier M, Bigelow C et al (2002) Recognition of osteoporosis by primary care physicians. Am J Public Health 92(2): 271-273

16. Agency for Healthcare Research and Quality. Plan-Do-Study-Act (PDSA) Cycle. https://innovations.ahrq.gov/qualitytools/plan-dostudy-act-pdsa-cycle. Accessed 10 April 2019

17. Yu M, Suh J, Nedley A, et al (2018) Fracture Liaison Service at Loma Linda University: why there is a real need. Poster presented at: Osteoporosis Symposium: Innovations in Bone Health Care. Loma Linda, CA

18. Barton DW, Behrend JC, Carmouche JJ (2019) Rates of osteoporosis screening and treatment following vertebral fracture. Spine J 3:411-417

19. Nguyen ET, Posas-Mendoza T, Siu AM et al (2018) Low rates of osteoporosis treatment after hospitalization for hip fracture in Hawaii. Osteoporos Int 8:1827-1832

20. Abrahamsen B, Skjødt MK, Vestergaard P (2019) Hip fracture rates and time trends in use of anti-osteoporosis medications in Denmark for the period 2005 to 2015: missed opportunities in fracture prevention. Bone 120:476-481
21. Hayes BL, Curtis JR, Laster A et al (2010) Osteoporosis care in the United States after declines in reimbursements for DXA. J Clin Densitom 4:352-360

22. Jose D, Torralba K, Downey C et al (2018) Fracture Liaison Service in an open health system: outcomes and challenges [abstract]. Arthritis Rheum 70(suppl 10)

23. Lafian A, Suh J, Torralba K (2017) Postmenopausal Breast Cancer Patients on Aromatase Inhibitor Therapy andTheir Increased Risk of Fragility Fracture [abstract]. Arthritis Rheumatol 69(suppl 10)

24. Accreditation Council for Graduate Medical Education. ACGME Common Program Requirements (Fellowship). https://www. acgme.org/Portals/0/PFAssets/ProgramRequirements/ CPRFellowship2019.pdf. Accessed 17 April 2019.

25. Torralba K, Yu M, Wang CB et al (2016) Interdisciplinary Osteoporosis Clinical Working Group and Fracture Liaison Service at Loma Linda University Medical Center (LLUMC): laying the Groundwork [abstract]. Arthritis Rheum 68(suppl 10)

26. Jose D, Downey C, Torralba K (2018) LLUMC Fracture Liaison Service: Tracking outcomes. Poster presented at: Loma Linda University Medical Center Annual Postgraduate Convention. Loma Linda, California

27. Kolb DA (2004) Experiential learning: experience as the source of learning and development. Englewood Cliffs, NJ

28. Yardley S, Teunisson PW, Dornan T (2012) Experiential Learning: AMEE Guide No. 63. Med Teach 34(2):e102-e115

29. Brown J, Chapman T, Graham D (2007) Becoming a new doctor: a learning or survival exercise? Med Educ 41:653-660

30. Collins A (2006) Cognitive apprenticeship. In: Sawyer RK (ed) Cambridge Handbook of the Learning Sciences. Cambridge University Press, Cambridge

31. Butler BA, Butler CM, Peabody TD (2019) Cognitive apprenticeship in orthopedic surgery: Updating a classic educational model. J Surg Educ 76:931-935

Publisher's note Springer Nature remains neutral with regard to jurisdictional claims in published maps and institutional affiliations. 